\title{
Descripción de las huellas de camélidos y félidos de la localidad Pie de Vaca, Cenozoico Tardío de Puebla, centro de México y algunas consideraciones paleobiológicas
}

\author{
Miguel Angel Cabral-Perdomo, Víctor Manuel Bravo-Cuevas, Alexis Pérez-Pérez, \\ Norberto García-Cabrera
}

\begin{abstract}
Miguel Angel Cabral-Perdomo
mcabralperdomo@gmail.com

Víctor Manuel Bravo-Guevas

Museo de Paleontología, Área Académica de Biología, Universidad Autónoma del Estado de Hidalgo, Ciudad del Conocimiento, Carretera Pachuca-Tulancingo, Km. 4.5, Colonia Carboneras, CP 42184, Mineral de la Reforma, Hidalgo, México.
\end{abstract}

\section{Alexis Pérez-Pérez}

Norberto García-Cabrera

Universidad Autónoma del Estado de Hidalgo, Ciudad del Conocimiento, Carretera Pachuca-Tulancingo, Km. 4.5, Colonia Carboneras, GP 42184, Mineral de la Reforma, Hidalgo, México.

BOL. SOC. GEOL. MEX. 2018

VOL. 70 NO. 2

P. $397-416$

http://dx.doi.org/10.18268/BSGM2018v70n2a9

Manuscrito recibido: Noviembre 1, 2017.

Manuscrito corregido: Enero 1, 2018.

Manuscrito aceptado: Enero 12,2018.

\section{RESUMEN}

La localidad Pie de Vaca en el estado de Puebla destaca por su importante abundancia de huellas fósiles pertenecientes a mamíferos, entre las cuales las más representativas han sido referidas a camélidos y félidos. E propósito del presente estudio fue la caracterización formal de este conjunto de huellas y comentar algunos aspectos paleobiológicos relacionados con el tamaño, velocidad y modalidad de progresión de los productores del registro icnofosilífero. La muestra consistió en 233 huellas: 154 pertenecen a nueve rastros producidos por camélidos y 79 a tres rastros por félidos. La caracterización de las huellas se realizó mediante la comparación de su tamaño y morfología con otras producidas por taxones fósiles y recientes. Las impresiones de camélidos, con base en su forma y tamaño, fueron designadas a la icnoespecie Lamaichnum guanicoe, ya que muestran el patrón morfológico típico de este grupo de artiodáctilos. Se sugiere que fueron producidas por algún integrante del género Hemiauchenia, el cual corresponde al camélido más común del Cenozoico tardío de México. Por su parte, las huellas de félidos únicamente fueron referidas a la morfofamilia Felipedidae debido a la mala preservación de sus caracteres icnotaxonómicos; sin embargo, al ser más grandes que huellas de los icnogéneros Felipeda, Pycnodactylopus, Pumaeichnum y Mitsupes, se sugiere que fueron producidas por alguna forma de talla mediana a grande, por ejemplo un macairodontino o un panterino. El modo de progresión de ambos productores corresponde con el de organismos con caminata relativamente rápida que se desplazan a una velocidad inferior a los $4 \mathrm{~m} / \mathrm{s}$. La dirección y número de individuos de los rastros de camélidos, son indicativos de un comportamiento gregario, probablemente asociado a cierta organización social. En el caso de los félidos se propone un comportamiento solitario o en pareja.

Palabras clave: icnita, camélidos, félidos, paso, zancada, progresión.

\section{ABSTRACT}

The Pie de Vaca locality in the State of Puebla stands out for its important abundance of fossil mammal footprints, among them, the most representatives have been referred to camelids and felids. The purpose of the present study was the formal characterization of this set of footprints and comment on some paleobiological aspects related to their size, speed, and mode of progression of the track-makers. The sample consists of 233 footprints including 154 referable to nine trackways produced by camelids and 79 referable to three trackways produced by felids. The characterization of the tracks was made by comparing their size and morphology with others produced by fossil and recent taxa. The impressions of camelids, based on their shape and size, were designated to the icnospecies Lamaichnum guanicoe because they show the typical morphological pattern of this group of artiodactyls. It is suggested that they were produced by some member of the genus Hemiauchenia, which corresponds to the most common camelid of the Late Cenozoic of Mexico. On the other hand, the tracks of felids were only referred to the morphofamily Felipedidae due to the bad preservation of its ichnotaxonomic characters; however, being larger than traces of the ichnogenera Felipeda, Pycnodactylopus, Pumaeichnum and Mitsupes, it is suggested that they were produced by some form of medium to large size, a machairodontid or a pantherine, for instance. The mode of progression of both producers corresponds to that of relatively fast walking organisms that move at a speed lower than $4 \mathrm{~m} / \mathrm{s}$. The direction and number of individuals of camelids trackways, are indicative of gregarious behavior, probably associated with a certain social organization. In the case of felids, solitary or couple behavior is proposed.

Keywords: ichnite, camelids, felids, pace, stride, progression. 


\section{Introducción}

En México, el registro paleoicnológico de vertebrados terrestres es relativamente abundante y diverso; la mayor parte de la información generada al respecto corresponde a huellas del Mesozoico que fueron producidas por dinosaurios, reptiles voladores, cocodrilomorfos, tortugas y aves (Rodríguez-de la Rosa et al., 2004). Por otro lado, los registros del Cenozoico de icnitas son más bien escasos: una localidad en Sinaloa, una en Durango, dos en Jalisco, una en Coahuila y cuatro en Puebla. En estos registros se han reportado huellas de aves y diversos mamíferos (Rodríguez-de la Rosa et al., 2004; Lockley y Delgado, 2007; Lockley y Bishop, 2014).

Sin duda, la localidad cenozoica con el mayor número de icnitas referidas para mamíferos que se conoce hasta el momento en México es Pie de Vaca, en el municipio de Tepexi de Rodríguez, estado de Puebla, la cual es conocida por los moradores del área desde hace más de 100 años. En esta localidad se pueden reconocer nueve rastros de camélidos, tres rastros de félidos, un rastro parcial de un artiodáctilo pequeño, huellas aisladas de aves y el tafoglifo (impresión del cadáver de un animal sensu Sarjeant, 1975: 284) de un flamenco (Lockley, 1991; Cabral-Perdomo, 1995, 2000, 2013). Las impresiones de los camélidos y félidos son las más representativas y abundantes en el sitio.

Si bien es cierto que el registro icnológico de Pie de Vaca se ha mencionado en otros trabajos (CabralPerdomo, 1995, 2000, 2013; Rodríguez-de la Rosa et al., 2004; Beraldi-Campesi et al., 2006), no se ha realizado su análisis icnológico. El presente trabajo tiene como propósito establecer la caracterización icnotaxonómica formal de las huellas de camélidos y félidos de la localidad Pie de Vaca, así como establecer algunas consideraciones paleobiológicas relacionadas con el tamaño, modo de progresión, velocidad y comportamiento de los individuos que las generaron.

\section{2. Área de estudio}

La localidad Pie de Vaca se localiza $3 \mathrm{~km}$ al noreste del centro de Tepexi de Rodríguez, Puebla, entre las coordenadas $18^{\circ} 35^{\prime} 24^{\prime \prime} \mathrm{N}$ y $97^{\circ} 55^{\prime} 08^{\prime \prime} \mathrm{W}$ (Figura 1). Esta unidad se denomina acríticamente formación Pie de Vaca, la cual se asocia a depósitos de origen lacustre (Beraldi-Campesi et al., 2006). En el Léxico Estratigráfico de México del Servicio Geológico Mexicano, la denominación de Pie de Vaca como unidad estratigráfica es informal (Juárez-Arriaga, 2008), dado que no cumple con los artículos necesarios propuestos por el Código de Nomenclatura Estratigráfico de Norteamérica para recibir el nombre de una unidad litoestratigráfica formal (Artículos 3-16 en North American Commission on Stratigraphic Nomenclature, 2005). Con base en esto, en el presente estudio, el nombre Pie de Vaca se usa de manera informal para referirse a la secuencia portadora de las impresiones.

En términos generales, la formación Pie de Vaca está compuesta por una sucesión de caliza micrítica de color que varía de anaranjado amarillento pálido (10YR 8/6) a anaranjado grisáceo (10YR 7/4) (Rock-Color Chart Committee, 2009). Tiene una estratificación delgada a mediana $(1-30 \mathrm{~cm})$, bandas de sílice e intraclastos de caliza, pedernal y roca volcánica, las cuales descansan en discordancia erosional sobre la Formación Tlayúa. La secuencia continúa con una serie alternante de grava, arena, limo y arcilla, seguidos de un conglomerado polimíctico bien compactado con fragmentos de caliza, pedernal, cuarzo y rocas volcánicas de composición variable (Pantoja-Alor et al., 1989) (Figura 2). La edad de esta unidad es controversial debido a que el registro de huellas 

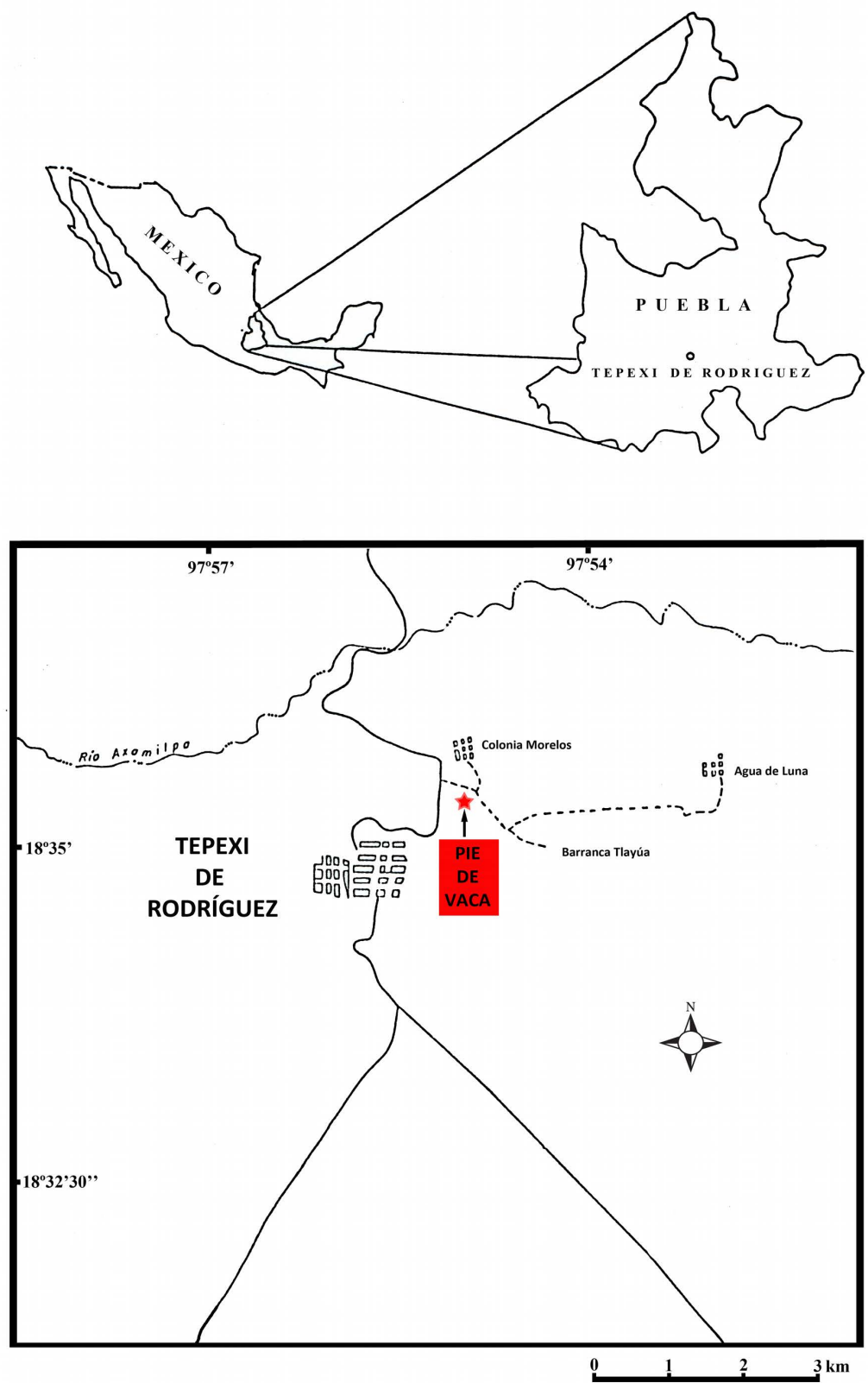

Figura 1 Mapa índice que muestra la localización de la localidad Pie de Vaca en la porción centro-meridional del estado de Puebla, centro de México.

de mamíferos sugiere una edad del Neógeno - Pleistoceno (Applegate et al., 1984; CabralPerdomo, 1995), mientras que evidencia palinológica recuperada de localidades cercanas a Pie de Vaca sugiere una edad más antigua del Paleógeno (Eoceno - Oligoceno) (Martínez-Hernández y Ramírez-Arriaga, 1999).
Precisar la edad de la unidad portadora está fuera del alcance del presente estudio; sin embargo, considerarla como parte del Paleógeno es poco probable si se toman en cuenta los siguientes hechos: (1) el tamaño de los camélidos que produjeron los rastros de Pie de Vaca no es consistente con el de animales del Paleógeno, dado que durante ese periodo 


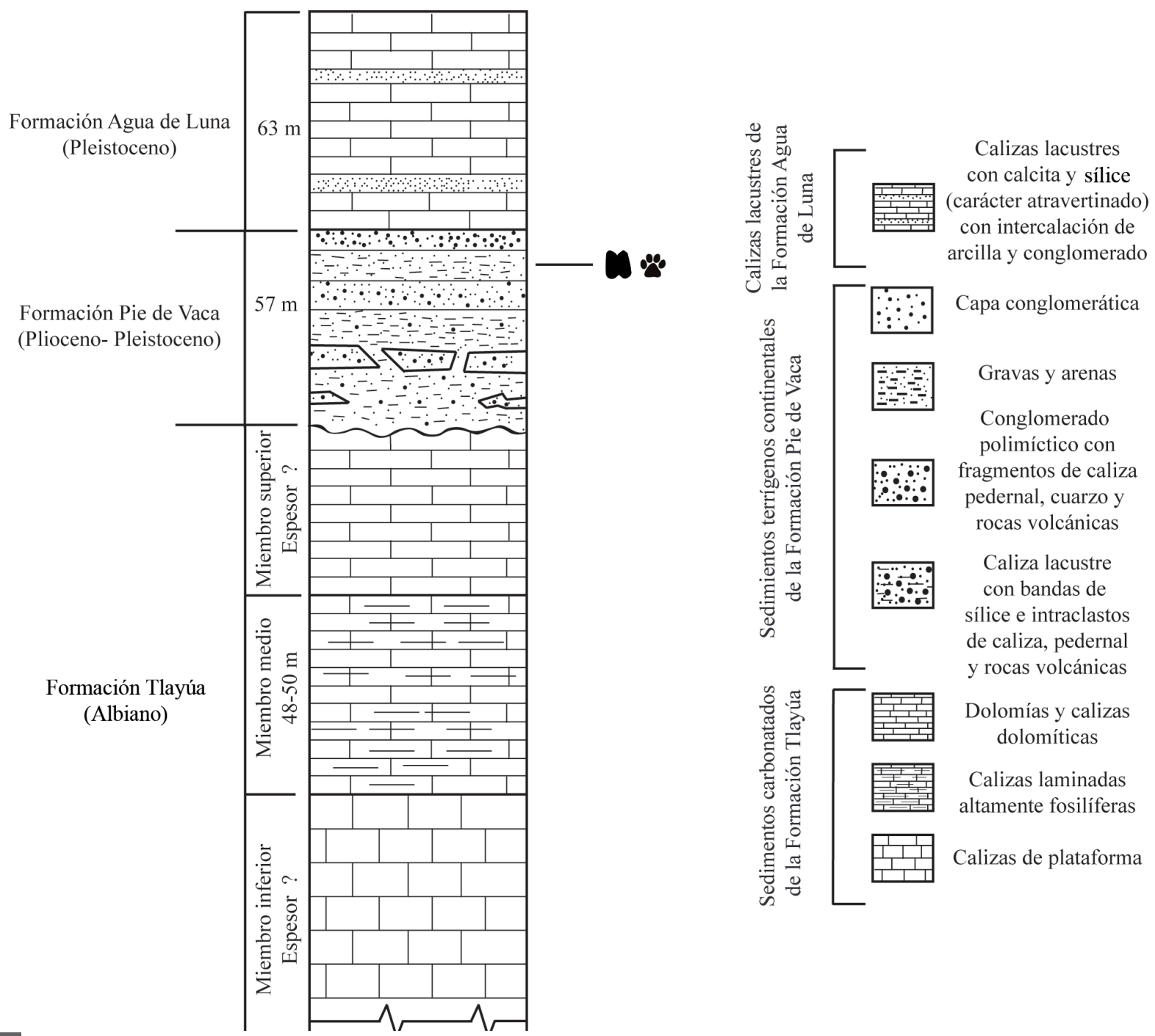

Figura 2 Columna estratigráfica del área de Tepexi de Rodríguez, Puebla (modificado de Cabral-Perdomo, 1995). Se indica el nivel portador de las huellas de camélidos y félidos de la localidad Pie de Vaca.

tuvieron un tamaño semejante al de una gacela; (2) el félido norteamericano más antiguo corresponde a Pseudaelurus, un inmigrante euroasiático del Mioceno (Prothero, 2006); (3) huellas pertenecientes a proboscídeos también se han reportado en estratos referibles a la formación Pie de Vaca, en la localidad Pochote - Elefante (Cabral-Perdomo, 1995) y se sabe que los primeros representantes de ese grupo llegaron a Norteamérica durante el Mioceno (Prothero, 2006). Aunado a esto, la configuración de las huellas y el tamaño relativamente grande de los organismos que produjeron el registro icnológico de Pie de Vaca es consistente con lo que se observa en los camélidos y félidos del Plioceno - Pleistoceno de Norteamérica (JiménezHidalgo et al., 2015). En este trabajo se considera que la edad más probable de la unidad portadora corresponde con este segmento geocronológico, como previamente algunos autores han indicado (Cabral-Perdomo, 1995, 2013; Jiménez-Hidalgo et al., 2015). El afloramiento icnofosilífero en el que se encuentran las huellas y rastros de camélidos (en lo sucesivo RG) y de félidos (en lo sucesivo RF) abarca una extensión de alrededor de $190 \mathrm{~m}^{2}$ (Figura 3A). En el mismo estrato portador de los fósiles se encontraba el tafoglifo de un flamenco, 

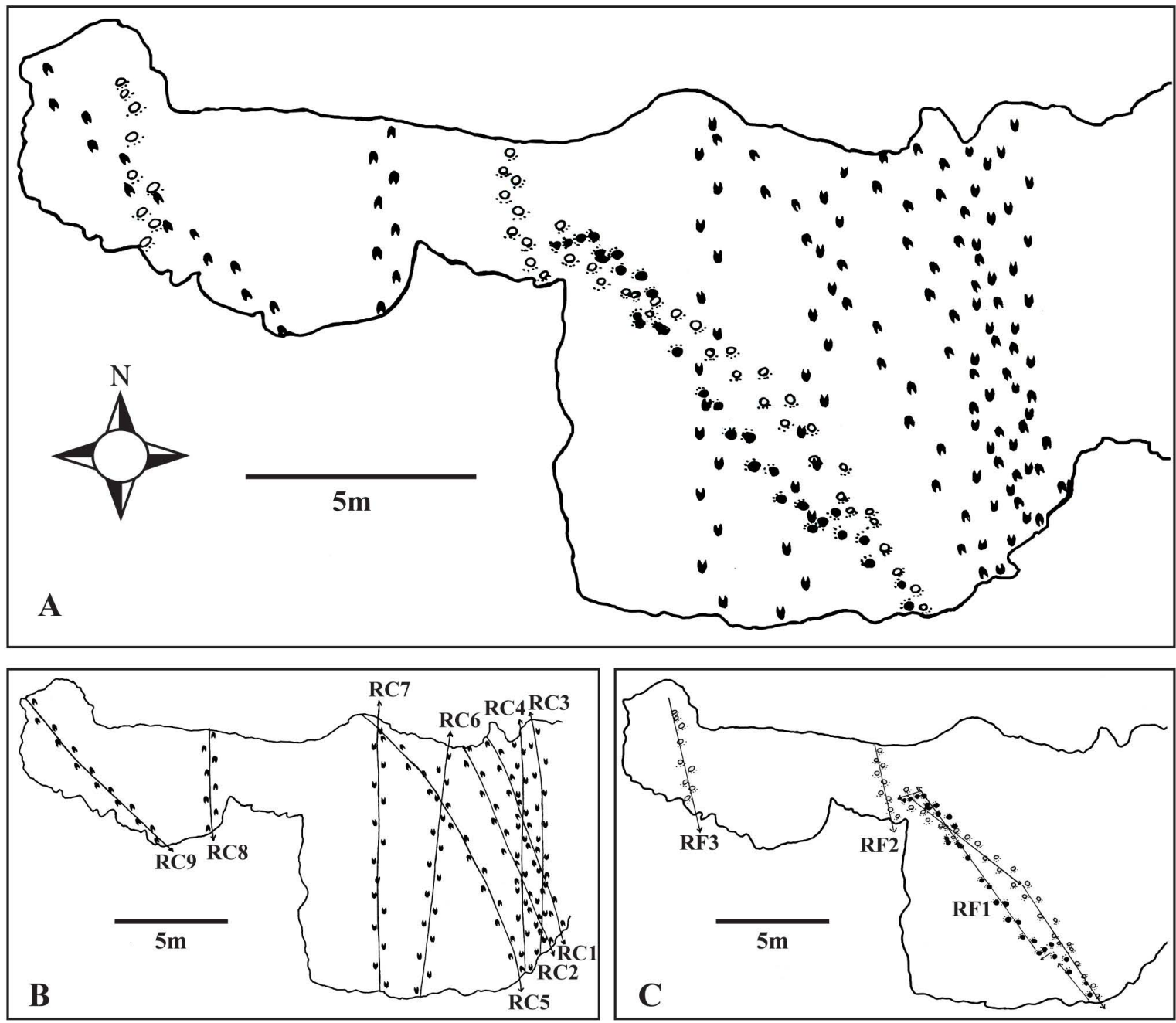

Figura 3 Distribución espacial de (A) las huellas de camélidos y félidos de Pie de Vaca, Cenozoico tardío de Puebla, centro de México. Se incluye la dirección de progresión de los individuos que generaron (B) los rastros de los camélidos (RC) y (C) los rastros de los félidos (RF). Barra de escala $=5$ metros.

el cual fue recolectado debido a que fue objeto de vandalismo y trasladado al Museo de Geología de la Universidad Nacional Autónoma de México.

\section{Materiales y Métodos}

La muestra considerada en este estudio incluye un total de 233 huellas: 154 de camélidos organizadas en nueve rastros y 79 de félidos organizadas en tres rastros.

Las huellas y los rastros se midieron con una cinta métrica flexible directamente en el afloramiento durante los meses de mayo y junio de 1994, como parte del proyecto de tesis de licenciatura del primer autor (Cabral-Perdomo, 1995). La estadística univariada (promedio, desviación estándar y rango observado) de las medidas se estimó usando el programa de estadística PAST para MacOS (Hammer et al., 2001). Las medidas están expresadas en $\mathrm{cm}$.

\subsection{DIMENSIONES ESTIMADAS}

En cada una de las huellas atribuidas a camélidos, se estimaron las siguientes medidas (Figura 4A): longitud y ancho internos de la huella; longitud 

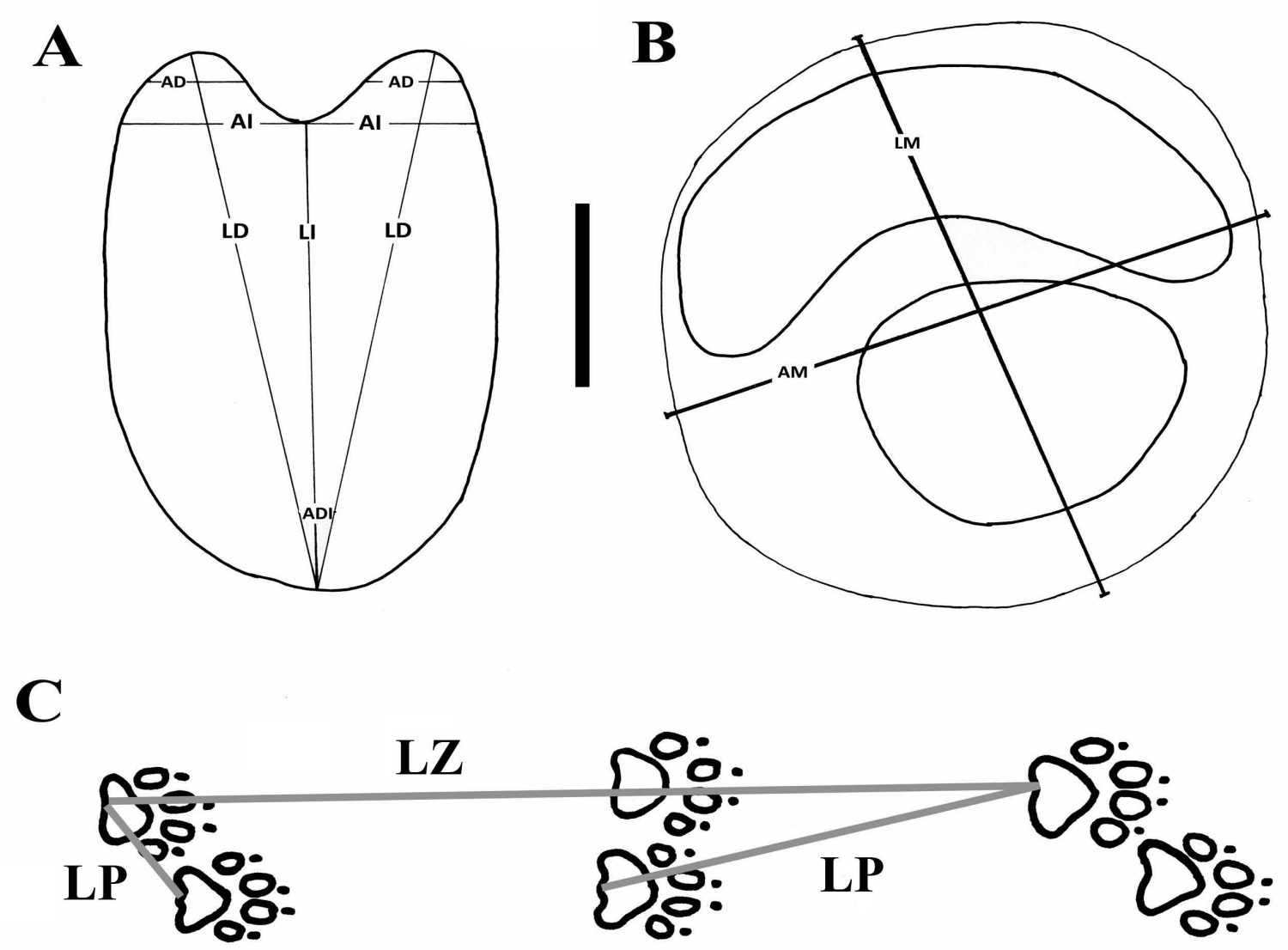

Figura 4 Esquemas de huellas de (A) camélido y (B) félido de Pie de Vaca, en los que se indican las dimensiones estimadas, así como un diagrama de un rastro de Canis familiaris (C) en el que se indican la longitud del paso (LP) y la longitud de la zancada (LZ). Abreviaturas: Al, Ancho interno de la huella; ADI, Ángulo Interdigital; AD, Ancho de los dígitos III y IV; AM, Ancho máximo de la huella; LI, Longitud interna de la huella; LD, Longitud de los dígitos III y IV; LM, Longitud máxima de la huella. Barra de escala $=5 \mathrm{~cm}$.

y ancho de cada dígito; y ángulo interdigital. Por otro lado, en cada una de las huellas atribuidas a félidos se estimó el ancho y la longitud máximos (Figura 4B).

En todos los rastros se estimó la longitud del paso y la zancada (Figura 4C), de acuerdo con lo propuesto por Sarjeant $(1975,1989)$.

\subsection{IDENTIDAD DE LOS PRODUGTORES DE LAS ICNITAS}

Con la finalidad de establecer la identidad de los productores potenciales de las huellas, se llevó a cabo una comparación del tamaño y morfología de las icnitas estudiadas con huellas producidas tanto por taxones extintos como por actuales, previamente reportados en la literatura (Aramayo y de Bianco, 1987; Remeika, 2001; Lucas y Hunt, 2007; Rodríguez-de la Rosa et al., 2007; PérezLorente et al., 2009; Diedrich, 2011; Aramayo et al., 2015).

\subsection{ESTIMAGIÓN DEL TAMAÑO Y LA VELOGIDAD}

La estimación del tamaño de los productores de las huellas se fundamenta en la correlación positiva entre la longitud de una huella con la altura de las extremidades de un mamífero bípedo o cuadrúpedo en reposo (Alexander, 1976; Thulborn, 1990; Henderson, 2003). Para tal efecto, se utilizó el 
método morfométrico de Alexander (1976) modificado por Thulborn (1990). En el presente estudio se describe con base en la siguiente ecuación:

$$
\mathrm{AE}=\mathrm{X} * \mathrm{~L}(\text { Ecuación } 1)
$$

AE corresponde a la altura promedio de las extremidades (anteriores y posteriores), L corresponde a la longitud promedio de las huellas y X se refiere a la proporción $\mathrm{AE} / \mathrm{L}$.

En el caso de los camélidos, la proporción AE/L se estableció tomando como referencia el promedio de la altura de las extremidades anteriores y posteriores $(\mathrm{AE}=97.9 \mathrm{~cm})$ (Bonacic y Franklin, 2006; Tabla 1 en Van der Sluijs et al., 2010: 292), así como la longitud promedio de huellas $(\mathrm{L}=10.8$ cm) (Tabla 3 en Remeika, 2001: 70) pertenecientes a la llama Lama glama. Para los félidos se llevó a cabo el mismo procedimiento, usando como referencia el promedio de la altura de las extremidades anteriores y posteriores $(\mathrm{AE}=120 \mathrm{~cm})($ Packer, 2006), así como la longitud promedio de huellas (L $=15.81 \mathrm{~cm}$ ) (fig. 75.3c en Liebenberg, 1990: 179) pertenecientes al león africano Panthera leo. Con base en los datos disponibles, la estimación de la altura promedio de las extremidades a partir de la longitud de la huella se determinó mediante las fórmulas $\mathrm{AE}=9 \mathrm{~L}$ y $\mathrm{AE}=7.6 \mathrm{~L}$ para camélidos y félidos respectivamente.

La velocidad de los productores de las huellas se estimó con base en la ecuación de Alexander (1976, 2006) como sigue:

$$
\mathrm{V}=0.25 \mathrm{~g}^{0.5} * \mathrm{LZ}^{1.67} * \mathrm{AE}^{-1.17} \text { (Ecuación 2) }
$$

Donde $\mathrm{V}$ corresponde a la velocidad, g es la aceleración de la gravedad $\left(9.8 \mathrm{~m} / \mathrm{s}^{2}\right)$ y LZ corresponde a la longitud promedio de la zancada. La velocidad estimada se expresa en m/s.

El modo de progresión de los camélidos y félidos de Pie de Vaca, se estableció mediante la proporción entre la longitud promedio de la zancada y la altura promedio de las extremidades (LZ/AE), dado que en términos generales, se sabe que un mamífero terrestre cambia de caminar a trotar/ correr cuando esta proporción alcanza un valor de aproximadamente 2.0 (Alexander, 1976). Con base en Thulborn (1982) y Thulborn y Wade (1984) se consideraron los siguientes modos de progresión: caminar $(\mathrm{LZ} / \mathrm{AE}<2.0)$, $\operatorname{trotar}(2.0<\mathrm{LZ} / \mathrm{AE}<$ 2.9) y correr (LZ/AE > 2.9).

La dirección de progresión de los individuos que generaron los rastros se determinó con base en la posición del norte magnético del Plioceno Pleistoceno, la cual es prácticamente indistinguible de la actual (García-Ruiz et al., 2016).

\section{Icnología Sistemática}

División Vertebratichnia Vialov, 1966

Clase Mammalipedia Vialov, 1966

Orden Artiodactipedia Vialov, 1966

Morfofamilia Pecoripedia Remeika et al., 1995

Icnogénero Lamaichnum Aramayo y de Bianco, 1987

Lamaichnum guanicoe Aramayo y de Bianco, 1987

Material referido. 154 huellas organizadas en 9 rastros, mismas que se encuentran en la localidad Pie de Vaca.

Descripción. Huellas conservadas en epirelieve cóncavo, subdigitígradas, de forma subovalada, longitud promedio de $13.2 \mathrm{~cm}$ y ancho promedio de $10.5 \mathrm{~cm}$, con un ángulo interdigital promedio de $29.6^{\circ}$ (Tabla 1). Las huellas muestran una morfología homogénea, lo que dificulta diferenciar las huellas producidas por las extremidades anteriores de las producidas por las posteriores (Homopodia). En términos generales, se distinguen por presentar impresiones dactilares subiguales en tamaño; dos marcas paralelas de uñas en punta separadas en la parte anterior; la región posterior de estas dos marcas confluye medialmente en una forma acorazonada y no se aprecia un surco medial (= septo interdigital); los bordes laterales son más o menos rectos y paralelos, mientras que el borde posterior es ligeramente convexo (Figura $5 \mathrm{~A})$. 


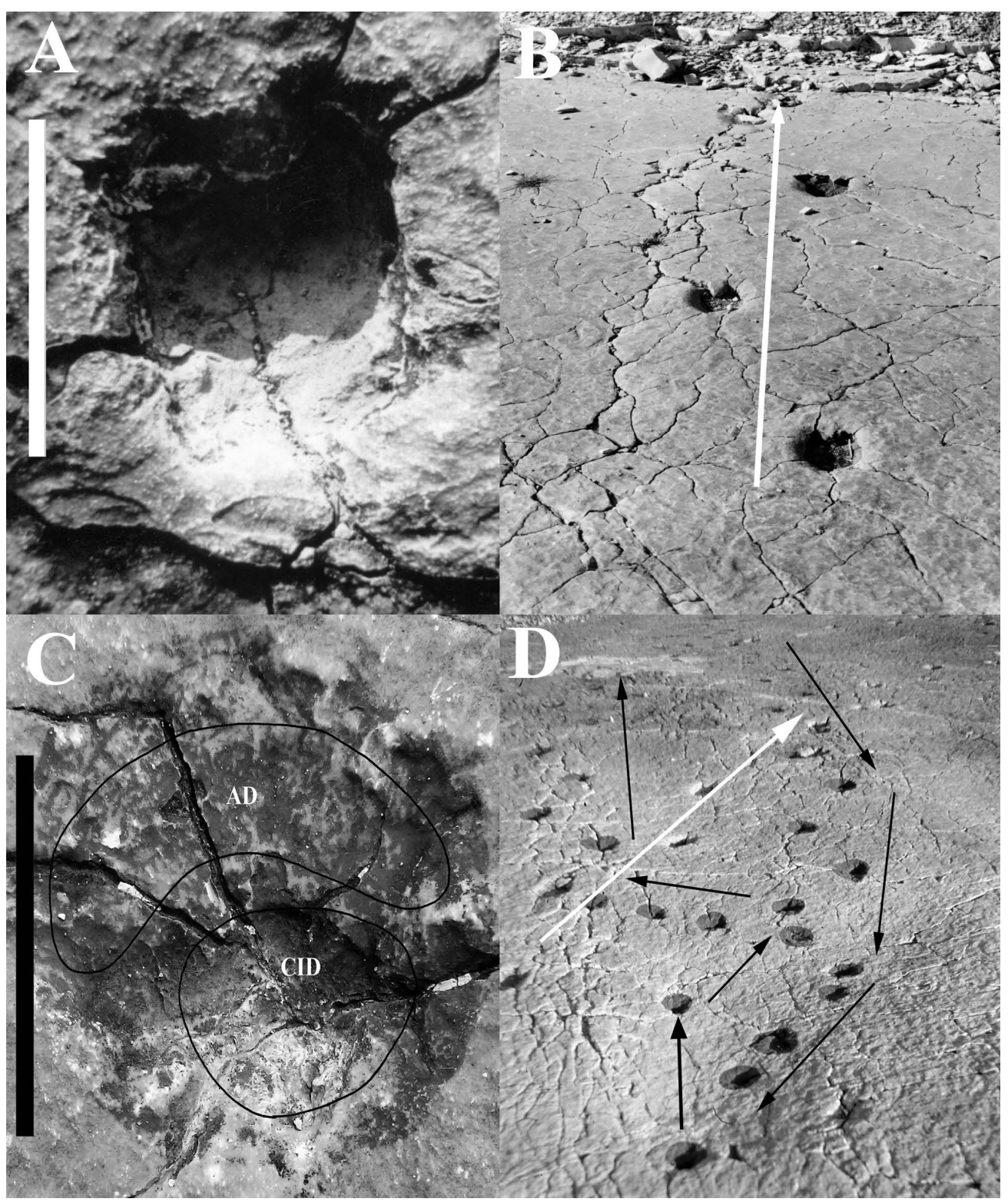

Figura 5 Huellas y rastros de Pie de Vaca: (A) Huella de camélido; (B) Secuencia de cuatro huellas del rastro 3 de camélido con la dirección de progresión indicada con la flecha blanca; (C) Huella de felino; (D) Vista parcial del afloramiento hacia el norte en donde se aprecia la progresión del felino del rastro 1 (flechas negras) y la progresión del camélido del rastro 6 (flecha blanca). Abreviaturas: AD, Arcada Digital; CID, Cojinete Interdigital. Barra de escala $=10 \mathrm{~cm}$.

Para cada uno de los nueve rastros, la longitud de paso promedio es de $69 \mathrm{~cm}$ y la longitud de zancada promedio es de $253 \mathrm{~cm}$ (figuras 3B y 5B, Tabla 1).

Discusión. Las ligeras diferencias en los bordes anterior y posterior de las huellas, así como la ausencia del surco interdigital, representan variación extramorfológica asociada a la interacción de pies-manos en un sustrato relativamente suave y con cierta humedad, así como alteración postdiagenética por intemperismo y erosión. La variación extramorfológica observada en huellas de camélidos es amplia y común (Lucas y Hunt, 2007).

De acuerdo a Lucas y Hunt (2007), a partir del Mioceno y hasta el Pleistoceno, los camélidos adquirieron una estructura podial estereotípica, lo 
Tabla 1. Promedio, desviación estándar y rango observado (entre paréntesis) de los parámetros evaluados en las huellas de camélidos de Pie de Vaca, Cenozoico tardío de Puebla, centro de México. Abreviaturas: LI, longitud interna de la huella; Al, ancho interno de la huella; LDIII, longitud del dígito III; LDIV, longitud del dígito IV; ADIII, ancho del dígito III; ADIV, ancho del dígito IV; ADI, ángulo interdigital; LP, longitud de paso; LZ, longitud de la zancada; N, número de huellas. Las medidas están en cm a excepción del ángulo interdigital.

\begin{tabular}{|c|c|c|c|c|c|c|c|c|c|c|}
\hline Rastro & $\mathrm{N}$ & LI & AI & LDIII & LDIV & ADIII & ADIV & ADI & LP & LZ \\
\hline $\mathrm{RC} 1$ & 17 & $\begin{array}{l}13.14 \pm 1.15 \\
(10.5-15.5)\end{array}$ & $\begin{array}{c}10.58 \pm 0.75 \\
(9.5-12)\end{array}$ & $\begin{array}{c}14.93 \pm 0.77 \\
(13.5-16)\end{array}$ & $\begin{array}{c}15.15 \pm 0.96 \\
(13-17)\end{array}$ & $\begin{array}{c}4.63 \pm 0.78 \\
(2.5-6)\end{array}$ & $\begin{array}{c}5.09 \pm 0.61 \\
(4-6)\end{array}$ & $\begin{array}{l}29.6^{\circ} \pm 3.4 \\
\left(20^{\circ}-35^{\circ}\right)\end{array}$ & $\begin{array}{l}68 \pm 2.98 \\
(60-71)\end{array}$ & $\begin{array}{c}253.15 \pm 6.86 \\
(240-261)\end{array}$ \\
\hline $\mathrm{RC} 2$ & 16 & $\begin{array}{c}13.21 \pm 1.21 \\
(11-15.5)\end{array}$ & $\begin{array}{c}10.31 \pm 0.35 \\
(10-11)\end{array}$ & $\begin{array}{c}14.50 \pm 1.06 \\
(12-16)\end{array}$ & $\begin{array}{l}14.26 \pm 0.77 \\
(12.5-15.5)\end{array}$ & $\begin{array}{c}4.63 \pm 0.39 \\
(4-5)\end{array}$ & $\begin{array}{c}4.70 \pm 0.56 \\
(3.5-5.5)\end{array}$ & $\begin{array}{l}29^{\circ} \pm 3.87 \\
\left(25^{\circ}-35^{\circ}\right)\end{array}$ & $\begin{array}{c}69.3 \pm 3.08 \\
(63-74)\end{array}$ & $\begin{array}{c}259.91 \pm 3.14 \\
(254-265)\end{array}$ \\
\hline $\mathrm{RC} 3$ & 18 & $\begin{array}{c}12.47 \pm 0.67 \\
-13.5)\end{array}$ & $\begin{array}{l}10.11 \pm 0.63 \\
(9.5-11.5)\end{array}$ & $\begin{array}{l}15.17 \pm 0.86 \\
(13.5-16.5)\end{array}$ & $\begin{array}{c}14.46 \pm 0.82 \\
(13-15.5)\end{array}$ & $\begin{array}{c}4.64 \pm 0.38 \\
(4-5)\end{array}$ & $\begin{array}{l}4.65 \pm 0.35 \\
\quad(4-5)\end{array}$ & $\begin{array}{l}29.1^{\circ} \pm 3.3 \\
\left(25^{\circ}-35^{\circ}\right)\end{array}$ & $\begin{array}{c}68.26 \pm 1.71 \\
(65-71)\end{array}$ & $\begin{array}{c}253.5 \pm 10.94 \\
(230-268)\end{array}$ \\
\hline $\mathrm{RC} 4$ & 19 & $\begin{array}{l}13.1 \pm 0.47 \\
(12.5-14)\end{array}$ & $\begin{array}{l}10.43 \pm 0.49 \\
(9.5-11.5)\end{array}$ & $\begin{array}{c}14.83 \pm 0.72 \\
(13.5-16)\end{array}$ & $\begin{array}{l}15.03 \pm 0.35 \\
(14.5-15.5)\end{array}$ & $\begin{array}{c}4.76 \pm 0.41 \\
(4-5.5)\end{array}$ & $\begin{array}{c}4.76 \pm 0.41 \\
(4-5.5)\end{array}$ & $\begin{array}{l}28.6^{\circ} \pm 3.5 \\
\left(25^{\circ}-35^{\circ}\right)\end{array}$ & $\begin{array}{c}68.36 \pm 2.30 \\
(63-71)\end{array}$ & $\begin{array}{c}249.46 \pm 8.7 \\
(231-264)\end{array}$ \\
\hline $\mathrm{RC} 5$ & 23 & $\begin{array}{c}13.5 \pm 0.58 \\
(12.5-14.5)\end{array}$ & $\begin{array}{c}10.13 \pm 0.45 \\
(9.5-11)\end{array}$ & $\begin{array}{c}14.58 \pm 0.59 \\
(13.5-16)\end{array}$ & $\begin{array}{c}14.60 \pm 0.45 \\
(14-15.5)\end{array}$ & $\begin{array}{c}4.0 \pm 0.45 \\
(3-4.5)\end{array}$ & $\begin{array}{l}4.0 \pm 0.39 \\
(3.5-4.5)\end{array}$ & $\begin{array}{l}29.6^{\circ} \pm 3.3 \\
\left(25^{\circ}-35^{\circ}\right)\end{array}$ & $\begin{array}{c}68.7 \pm 2.07 \\
(64-72)\end{array}$ & $\begin{array}{c}251.84 \pm 5.02 \\
(243-263)\end{array}$ \\
\hline RC6 & 17 & $\begin{array}{l}13.67 \pm 0.76 \\
(12.5-15.5)\end{array}$ & $\begin{array}{l}10.08 \pm 0.64 \\
(9.5-11.5)\end{array}$ & $\begin{array}{c}14.26 \pm 0.50 \\
(13.5-15)\end{array}$ & $\begin{array}{c}14.20 \pm 0.39 \\
(13.5-15)\end{array}$ & $\begin{array}{c}4.38 \pm 0.37 \\
(4-5)\end{array}$ & $\begin{array}{l}4.41 \pm 0.36 \\
\quad(4-5)\end{array}$ & $\begin{array}{l}29.7^{\circ} \pm 3.3 \\
\left(25^{\circ}-35^{\circ}\right)\end{array}$ & $\begin{array}{c}69.62 \pm 1.57 \\
(65-72)\end{array}$ & $\begin{array}{c}259.61 \pm 4.57 \\
(251-266)\end{array}$ \\
\hline $\mathrm{RC} 7$ & 18 & $\begin{array}{c}13.42 \pm 0.38 \\
(13-14)\end{array}$ & $\begin{array}{c}10.39 \pm 0.34 \\
(10-11)\end{array}$ & $\begin{array}{c}14.53 \pm 0.53 \\
(14-15.5)\end{array}$ & $\begin{array}{c}14.60 \pm 0.28 \\
(14-15)\end{array}$ & $\begin{array}{c}4.32 \pm 0.50 \\
(3.5-5)\end{array}$ & $\begin{array}{c}4.32 \pm 0.24 \\
(4.0-4.5)\end{array}$ & $\begin{array}{c}30^{\circ} \pm 2.7 \\
\left(25^{\circ}-35^{\circ}\right)\end{array}$ & $\begin{array}{c}70.20 \pm 1.57 \\
(67-72)\end{array}$ & $\begin{array}{c}266.6 \pm 3.98 \\
(260-271)\end{array}$ \\
\hline $\mathrm{RC} 8$ & 9 & $\begin{array}{l}13.16 \pm 0.35 \\
(12.5-13.5)\end{array}$ & $\begin{array}{c}10.4 \pm 0.39 \\
(10-11)\end{array}$ & $\begin{array}{c}14.38 \pm 0.33 \\
(14-15)\end{array}$ & $\begin{array}{c}14.4 \pm 0.39 \\
(14-15)\end{array}$ & $\begin{array}{c}4.2 \pm 0.44 \\
(3.5-5)\end{array}$ & $\begin{array}{c}4.16 \pm 0.35 \\
(3.5-4.5)\end{array}$ & $\begin{array}{l}28.3^{\circ} \pm 2.5 \\
\left(25^{\circ}-30^{\circ}\right)\end{array}$ & $\begin{array}{c}68.8 \pm 2.70 \\
(64-72)\end{array}$ & $\begin{array}{l}248 \pm 3.67 \\
(243-252)\end{array}$ \\
\hline RC9 & 17 & $\begin{array}{c}13.29 \pm 0.50 \\
(12.5-14)\end{array}$ & $\begin{array}{c}10.29 \pm 0.39 \\
(9.5-11)\end{array}$ & $\begin{array}{c}14.38 \pm 0.37 \\
(13.5-15)\end{array}$ & $\begin{array}{l}14.26 \pm 0.31 \\
(13.5-14.5)\end{array}$ & $\begin{array}{c}4.0 \pm 0.43 \\
(3.5-5)\end{array}$ & $\begin{array}{c}3.94 \pm 0.34 \\
(3.5-4.5)\end{array}$ & $\begin{array}{l}28.8^{\circ} \pm 3.3 \\
\left(25^{\circ}-35^{\circ}\right)\end{array}$ & $\begin{array}{c}68.59 \pm 2.16 \\
(64-71)\end{array}$ & $\begin{array}{c}248.30 \pm 8.46 \\
(229-258)\end{array}$ \\
\hline
\end{tabular}

que dificulta distinguir fehacientemente las huellas dejadas por diferentes géneros o especies de ese grupo de artiodáctilos. Estos autores proponen que las huellas de camélidos que abarcan dicho lapso sean asignadas únicamente al icnogénero Lamaichnum. Para estos autores, este icnotaxón está integrado por las icnoespecies L. guanicoe y L. macropodum, las cuales se diferencian prácticamente por el tamaño. Las huellas con una longitud de 4 a $15 \mathrm{~cm}$ se asignan a $L$. guanicoe y aquellas con una longitud de 16 a $20 \mathrm{~cm}$ se atribuyen a $L$. macropodum (Lucas y Hunt, 2007). Recientemente, (Aramayo et al., 2015) se ha propuesto una nueva icnoespecie dentro del icnogénero: L. tulipensis, misma que se caracteriza por tener heteropodia, por tener las impresiones de los dedos de las manos dirigidos hacia afuera y por tener dimensiones mayores a las de L. guanicoe. Sin embargo, Oliva y Arregui (2018) consideran que la morfología de estas huellas se debe a patologías en los individuos productores, por lo que sinonimizan a $L$. tulipensis con L. guanicoe. Las huellas de Pie de Vaca tienen una longitud promedio de $13 \mathrm{~cm}$, la cual está en el límite superior de las L. guanicoe y, por lo tanto, se asignan a esta icnoespecie.

La mayoría de las icnitas referidas para el país son de dimensiones que se encuentran en el rango de Lamaichnum guanicoe. De esta forma, en la localidad Las Pintas en el estado de Durango, Lockley y Delgado (2007) describen un rastro en cuyas huellas estimaron longitudes de 5 a $8 \mathrm{~cm}$ y ancho de 5 a $6 \mathrm{~cm}$. Los autores confirieron estos icnofósiles al icnogénero Lamaichnum sin pretender identificarlas a nivel específico.

En otro reporte de índole meramente geológico (Dávalos-Álvarez et al., 2007), se menciona la presencia de icnofósiles de camélidos en la unidad Sedimentos Lacustres Villa Alegría de la Formación Tehuacán en el estado de Puebla, pero no se describen formalmente. En la ilustración que presentan (fig. B 1 en Dávalos-Álvarez et al., 2007: 212) se puede apreciar que la morfología y 
Tabla 2. Promedio, desviación estándar y rango observado (entre paréntesis) de los parámetros evaluados en las huellas de félidos de Pie de Vaca, Cenozoico tardío de Puebla, centro de México. Abreviaturas: LM, longitud máxima de la huella; AM, ancho máximo de la huella; LP, longitud de paso; LZ, longitud de la zancada; N, número de huellas. Las medidas están en cm.

\begin{tabular}{|c|c|c|c|c|c|}
\hline Rastro & $\mathrm{N}$ & LM & AM & LP & LZ \\
\hline RF1 & 17 & $12.71 \pm 1.79$ & $12.54 \pm 1.63$ & $50.31 \pm 9.02$ & $191.36 \pm 3.06$ \\
& & $(8-20)$ & $(8-16)$ & $(35-60)$ & $(187-197)$ \\
\hline RF2 & 16 & $13.44 \pm 1.26$ & $12.33 \pm 1.47$ & $39.75 \pm 1.70$ & $154.4 \pm 7.33$ \\
& & $(11-15)$ & $(10-15)$ & $(38-42)$ & $(145-163)$ \\
\hline RF3 & 18 & $14.44 \pm 1.33$ & $13.5 \pm 1.95$ & $42 \pm 2.64$ & $167 \pm 7.07$ \\
& & $(13-17)$ & $(10-16)$ & $(40-45)$ & $(162-172)$ \\
\hline
\end{tabular}

dimensiones de las huellas de este sitio son comparables a las de Tepexi de Rodríguez, por lo que los autores consideran que la edad de la unidad portadora de las huellas de Tehuacán sería semejante a la de la localidad Pie de Vaca.

Un último reporte de icnofósiles de camélidos para el país es el publicado por Rodríguez-de la Rosa y Guzmán-Gutiérrez (2012), quienes reportaron huellas de Lamaichnum macropodum en la localidad de San Juan de Los Lagos en Jalisco. Las icnitas tienen en promedio $16 \mathrm{~cm}$ de longitud y $11.7 \mathrm{~cm}$ de ancho, dimensiones que caen dentro del rango propuesto por Lucas y Hunt (2007) para dicha icnoespecie.

Orden Carnivoripedida Vialov, 1966

Morfofamilia Felipedidae Remeika, 2001

Material reportado. 79 icnitas repartidas en tres rastros que continúan en la localidad Pie de Vaca.

Descripción. Huellas conservadas en epirelieve cóncavo, digitígradas, subredondeadas, longitud promedio de $13.5 \mathrm{~cm}$ y ancho promedio de 12.8 cm (Tabla 2). La forma general de las huellas es muy semejante (Homopodia) y, por lo tanto, es dificil distinguir entre impresiones manuales y podiales. Las huellas tienen una profundidad que varía de 1 a $3 \mathrm{~cm}$. En la parte posterior presentan un cojinete interdigital de forma subtriangular a semiredonda que en ocasiones es muy profundo, mientras que en la parte anterior se observa una depresión reniforme continua menos marcada que el cojinete interdigital y que corresponde al área en la que se dispone la arcada de los cojinetes dactilares. El borde anterior de dicha depresión es convexo y no se aprecian impresiones de garras o uñas (Figura 5C). En este conjunto se incluyen 79 huellas organizadas en tres rastros. La longitud promedio del paso es de $44 \mathrm{~cm}$ y la longitud promedio de la zancada es de $171 \mathrm{~cm}$ (figuras $3 \mathrm{C}$ y 5C-D, Tabla 2).

Discusión. La configuración de las huellas de félidos de Pie de Vaca es semejante a las impresiones incluidas en la morfofamilia Felipedidae, por su condición digitígrada, la configuración de la arcada de los dígitos, así como la forma y tamaño del cojinete interdigital (Remeika, 2001). Las huellas de félidos de Pie de Vaca tienen el doble de tamaño que las consideradas en Felipeda (longitud $\leq 5 \mathrm{~cm}$ ) y en Pumaeichnum (longitud $\approx 6 \mathrm{~cm}$ ), son un $40 \%$ más grandes que las de Pycnodactylopus (longitud $\approx 8 \mathrm{~cm}$ ) y un $25 \%$ más grandes que las de Mitsupes (longitud $\approx 10 \mathrm{~cm}$ ). Proponer un nuevo icnotaxón con base en el tamaño grande de las huellas de Pie de Vaca es poco pertinente. Aunado a esto, su pobre detalle en otros rasgos diagnósticos, tales como el tamaño, configuración y disposición de cada uno de los dígitos, limitan una evaluación y comparación completas de su patrón morfotípico. Por lo tanto, se prefirió identificarlas icnotaxonómicamente a nivel de la morfofamilia. En lo que respecta a la diversidad icnotaxonómica de félidos en Norteamérica, Sarjeant et al. (2002) reconocieron a los icnogéneros Pycnodactylopus y Felipeda en la morfofamilia Felipedidae, mientras que Remeika $(2001,2006)$ considera únicamente al icnogénero Pumaeichnum con tres icnoespecies $(P$. 
Tabla 3. Estimación de la altura promedio de las extremidades (AE), dirección de progresión (Dp), velocidad (V) y modo de progresión de los camélidos y félidos de Pie de Vaca, Cenozoico tardío de Puebla, centro de México. N, número de huellas. La proporción de la longitud de la zancada y altura de la extremidad (LZ/AE) se asoció a los siguientes modos de progresión: $\mathrm{LZ} / \mathrm{AE}<2.0=\mathrm{caminar}, 2.0<\mathrm{LZ} / \mathrm{AE}<$ $2.9=$ trotar, $\mathrm{LZ} / \mathrm{AE}>2.9=$ correr.

\begin{tabular}{|c|c|c|c|c|c|c|}
\hline Rastro & $\mathrm{N}$ & $\begin{array}{c}\mathrm{AE} \\
(\mathrm{cm})\end{array}$ & $\mathrm{Dp}$ & $\mathrm{V}(\mathrm{m} / \mathrm{s})$ & $\mathrm{LZ} / \mathrm{AE}$ & Progresión* \\
\hline \multicolumn{7}{|l|}{ Camélidos } \\
\hline $\mathrm{RC} 1$ & 17 & 118.32 & $22^{\circ} \mathrm{SE}$ & 3.03 & 2.13 & trote \\
\hline $\mathrm{RC} 2$ & 16 & 118.96 & $24^{\circ} \mathrm{SE}$ & 3.15 & 2.18 & trote \\
\hline $\mathrm{RC} 3$ & 18 & 112.25 & $\mathrm{~N}$ & 3.22 & 2.25 & trote \\
\hline $\mathrm{RC} 4$ & 19 & 117.9 & $\mathrm{~N}$ & 2.98 & 2.12 & trote \\
\hline RC5 & 23 & 121.5 & $31^{\circ} \mathrm{SE}$ & 2.91 & 2.07 & caminar-trote \\
\hline RC6 & 17 & 123.08 & $07^{\circ} \mathrm{NE}$ & 3.01 & 2.11 & trote \\
\hline $\mathrm{RC7}$ & 18 & 120.85 & $\mathrm{~N}$ & 3.23 & 2.2 & trote \\
\hline $\mathrm{RC} 8$ & 9 & 118.5 & $02^{\circ} \mathrm{SE}$ & 2.93 & 2.09 & caminar-trote \\
\hline $\mathrm{RC} 9$ & 17 & 119.64 & $43^{\circ} \mathrm{SE}$ & 2.91 & 2.07 & caminar-trote \\
\hline \multicolumn{7}{|l|}{ Félidos } \\
\hline RF1 & 61 & 96.6 & $36^{\circ} \mathrm{NO}-43^{\circ} \mathrm{SE}$ & 2.41 & 1.98 & caminar-trote \\
\hline RF2 & 9 & 102.14 & $10^{\circ} \mathrm{SE}$ & 1.57 & 1.51 & caminar \\
\hline RF3 & 9 & 109.74 & $12^{\circ} \mathrm{SE}$ & 1.66 & 1.52 & caminar \\
\hline
\end{tabular}

biancoi, P. milleri y P. stouti) del Plioceno temprano de California. Recientemente, Rodríguez-de la Rosa y Guzmán-Gutiérrez (2012), erigieron a la icnoespecie Mitsupes dugesi para identificar a un conjunto de huellas atribuidas a macairodontinos del Neógeno tardío de Jalisco, centro-norte de México. En el Mioceno de España y Rumania se describieron huellas de félidos referidas al icnogénero Felipeda con dos icnoespecies: F. lynxi y $F$ parvula (Anton et al., 2004).

\section{Consideraciones paleobiológicas del registro}

\subsection{AFINIDAD DEL PRODUGTOR DE LAS HUELLAS DE GAMÉLIDOS}

Las huellas de camélidos de Pie de Vaca son semejantes a las impresiones que dejan estos artiodáctilos en los siguientes rasgos: (1) digitígradas, (2) huellas de configuración ovoidal, (3) impresiones manuales y podiales con una morfología semejante, (4) ápices de los dígitos dirigidos anteriormente, así como (5) borde lateral de los dígitos convexo y borde medial recto (Aramayo y de Bianco, 1987; Sarjeant y Langston, 1994; Remeika, 2001; Lucas y Hunt, 2007).

El tamaño promedio de las huellas de Pie de Vaca es un 15\% mayor al de huellas atribuidas a Hemiauchenia sp. del Plioceno de California y aproximadamente un 20\% más grande al de las llamas actuales de la especie Lama glama (Remeika, 2001, 2006). Entre los camélidos más comunes del Cenozoico tardío de Norteamérica con un tamaño algo más grande que las llamas y guanacos actuales está Hemiauchenia (Hulbert y Webb, 2001; Lange, 2002). Por lo anterior, es probable que los productores de las huellas de camélidos de Pie de Vaca hayan sido integrantes de ese género. 


\subsubsection{TAMAÑO, VELOCIDAD Y MODO DE PROGRESIÓN}

La altura promedio de las extremidades de los camélidos de Pie de Vaca es relativamente homogénea, aproximadamente de $1.2 \mathrm{~m}$. El individuo que produjo el rastro 3 correspondería al más pequeño $(1.12 \mathrm{~m})$ y el más grande al del rastro 6 $(1.23 \mathrm{~m})$ (Tabla 3). Los rastros 1, 2, 5, 8 y 9 son paralelos, se dirigen hacia el sureste y están espaciados por una distancia que varía de $2.5 \mathrm{~m}$ a 12 m. Por su parte, los rastros 3, 4, 6 y 7 son paralelos, se dirigen al norte-noreste y están separados por una distancia promedio de $3 \mathrm{~m}$ (Figura 3B, Tabla 3). La velocidad promedio de los productores de los rastros es de $3.04 \mathrm{~m} / \mathrm{s}$. La proporción LZ/AE es en promedio de 2.13 y se asociaría a un cambio en el modo de progresión de caminar a trotar (Alexander, 1976; Thulborn, 1982) (Tabla 3).

La altura promedio de las extremidades de los camélidos de Pie de Vaca es mayor (en un 30\%) en comparación a la que alcanzan vicuñas y alpacas ( $c a .0 .85 \mathrm{~m}$ ), mientras que es ligeramente más grande (alrededor de un 10\%) a la de guanacos y llamas (ca. $1.10 \mathrm{~m}$ ) (Bonacic y Franklin, 2006; Van der Sluijs et al., 2010). Las llamas del género Hemiauchenia del Plioceno y Pleistoceno de Norteamérica son algo más grandes que una llama actual (Lange, 2002). Por lo anterior, es probable que los camélidos que generaron los rastros hayan sido representantes de ese género de lamines, el cual fue bastante común a lo largo del territorio nacional durante el Cenozoico tardío (Jiménez-Hidalgo y Carranza-Castañeda, 2010; Bravo-Guevas et al., 2012, 2016a).

La dirección de los rastros, los valores de velocidad y modalidad de progresión de los organismos que los produjeron, así como la profundidad de las huellas, son evidencia utilizada para establecer un comportamiento gregario a través de huellas (Castanera et al., 2012). La distribución y dirección de los rastros de camélidos de Pie de Vaca se asociaría a un grupo de cinco individuos que se dirigen hacia el sureste ( $R G$ 1, 2, 5, 8 y 9) y otro grupo de cuatro individuos que se desplazan hacia el norte-noreste (RC 3, 4, 6 y 7). Aunado a esto, el traslape que se observa entre los rastros 5 y 6 , así como entre los rastros 1 y 2 con los rastros 3 y 4 (Figura 3A y 3B), indica que los grupos propuestos deambularon por el sitio en momentos diferentes. Con base en la dirección de los rastros, el hecho de los organismos que los produjeron exhiben una velocidad y modo de progresión semejante, así como a la homogénea profundidad de las huellas $(\approx 4 \mathrm{~cm})$, se propone un comportamiento gregario que a su vez sugiere cierta interacción social.

Entre las especies actuales de llamas, solamente Lama guanicoe (guanaco) y Vicugna vicugna (vicuña) son salvajes y se distribuyen exclusivamente en Sudamérica (Lange, 2002); ambas especies muestran una marcada organización social y comportamiento territorial (Tomka, 1992). Los guanacos constituyen tres grupos sociales principales: (1) grupo familiar, (2) tropas de machos y (3) machos solitarios. Los grupos familiares están integrados por un macho adulto, el harem de cinco o seis hembras adultas, una o dos crías y un juvenil. Las tropas de machos están formadas por tres y hasta 60 jóvenes-adultos que no son territoriales. Los machos solitarios suelen ser individuos adultos maduros con o sin territorio (Raedeke, 1979; Pérez-Lorente et al., 2009; Franklin, 2011).

La poca variación en la altura promedio de las extremidades de los individuos que generaron los rastros de Pie de Vaca, sugiere un grupo de camélidos de tamaño semejante. Con base en el comportamiento gregario que testifica el registro icnofosilífero y asumiendo una organización social similar a la que se observa en los guanacos actuales, es probable que los rastros de camélidos de Pie de Vaca evidencien la presencia de tropas.

Como ya se ha mencionado, las huellas de camélidos de Pie de Vaca son muy homogéneas en tamaño y morfología, lo cual en la mayoría de los casos imposibilita distinguir las impresiones manuales de las podiales. Sin embargo, la sucesión de huellas que forman los rastros indica una alternancia de las extremidades (anterior/posterior) asociada a pasos bien distanciados que resulta en un patrón de distribución de las impresiones a manera de zigzag. Este patrón es muy semejante al que se produce cuando un animal cuadrúpedo 
está en canter (Alemán: Galopp) (fig. 2 D en Collins y Stewart, 1993: 335). El canter es un movimiento semejante al galope, aunque relativamente más lento, en el que las extremidades anterior derecha/ posterior izquierda se mueven juntas en una fase (Webb, 1972; Collins y Stewart, 1993; Kienapfel et al., 2014). La velocidad promedio de los camélidos de Pie de Vaca es mayor en comparación a la velocidad promedio de una llama o una alpaca al caminar, la cual es de $1.13 \mathrm{~m} / \mathrm{s}$ y $0.97 \mathrm{~m} / \mathrm{s}$ respectivamente (Pfau et al., 2011; Kienapfel et al., 2014). Con base en esto, se propone que los camélidos que generaron los rastros de Pie de Vaca estaban realizando un caminar rápido similar al canter.

\subsection{AFINIDAD DEL PRODUCTOR DE LAS HUELLAS DE FÉLIDOS}

La configuración de estas huellas es semejante a las impresiones de félidos en los siguientes rasgos: (1) digitígradas, (2) arcada de los dígitos semicircular y ubicada enfrente del cojinete interdigital, (3) cojinete interdigital que ocupa una buena parte de la huella, así como (4) impresiones de las garras usualmente ausentes (Remeika, 2001; Sarjeant et al., 2002).

Las huellas de félidos de Pie de Vaca son similares a las impresiones referidas a macairodontinos del Plioceno de Jalisco (Rodríguez-de la Rosa et al., 2007) y a las impresiones referidas al león de las cavernas Panthera spelea del Pleistoceno tardío del norte de Alemania (fig. 2 en Diedrich, 2011: 1885) en la presencia de un cojinete interdigital grande de forma subtriangular a semiredondeada, así como arcada de los dígitos con borde anterior convexo. En lo que respecta al tamaño, son más cercanas a las atribuidas al león de las cavernas de Alemania (longitud de aproximadamente $14 \mathrm{~cm}$ ) y relativamente más grandes que las de los macairodontinos de Jalisco (longitud de $10 \mathrm{~cm}$ ).

En el Plioceno de California se conoce un conjunto de huellas significativamente más pequeñas (longitud promedio de $6 \mathrm{~cm}$ ) en comparación con las de Pie de Vaca, las cuales se asociaron a las especies extintas Felis lacustris (félido semejante a un lince) y Acinonyx (Miracinonyx) studeri (chita semejante en tamaño a un puma) (figuras 8 y 9 en Remeika, 2001: 62 y 64 respectivamente) del Blancano Irvingtoniano del occidente de Norteamérica (Werderlin, 1985) y del Irvingtoniano temprano de California (Jefferson y Tejada-Flores, 1995), respectivamente.

El tamaño y morfología de las huellas de félidos de Pie de Vaca son indicativos de una forma de talla mediana a grande, tal vez un panterino o un macairodontino; sin embargo, la pobre definición de las huellas imposibilita precisar la identidad de sus productores.

\subsubsection{TAMAÑO, VELOCIDAD Y MODO DE PROGRESIÓN}

La altura promedio de las extremidades de los félidos es aproximadamente de $1 \mathrm{~m}$. El individuo que generó el RF 1 correspondería al más pequeño y el más grande al que generó el RF 3 (Tabla 3). El RF 1 tiene una dirección al noroeste y luego al sureste, así como se traslapa con los rastros 6 y 7 de camélidos. Los RF 2 y RF 3 son paralelos, dirigidos hacia el sureste y espaciados por unos $8 \mathrm{~m}$; en particular, el RF 2 se traslapa con el rastro 9 de camélidos (Tabla 3, Figura 3A y 3C). La velocidad promedio de los individuos que generaron los rastros es de $1.88 \mathrm{~m} / \mathrm{s}$. La proporción LZ/AE es en promedio de 1.67 y se asociaría a organismos en caminata; sin embargo, la determinada para el individuo que se asocia al rastro $1(\mathrm{LZ} / \mathrm{AE} \approx 2)$ sugiere un cambio en el modo de progresión de caminar a trotar (Alexander, 1976; Thulborn, 1982) (Tabla 3).

La altura promedio de las extremidades de los félidos de Pie de Vaca $(c a .1 .0 \mathrm{~m})$ es indicativa de organismos de talla mediana a grande (Kleiman y Eisenberg, 1973), es mayor en comparación con la de un puma (Puma concolor; $\mathrm{AE}=0.75 \mathrm{~m}$ ) (Currier, 1983), con la de un leopardo de las nieves (Uncia uncia; $\mathrm{AE}=0.60 \mathrm{~m}$ ) (Hemmer, 1972), con la de un leopardo africano (Panthera pardus; $\mathrm{AE}=$ $0.64 \mathrm{~m}$ ) (Stein y Hayssen, 2013) y con la de un chita (Acinonyx jubatus; $\mathrm{AE}=0.80 \mathrm{~m}$ ) (Krausman y Morales, 2005); asimismo, es algo más pequeña 
(en aproximadamente un $15 \%$ ) que la de un tigre (P. tigris) y un león africano (P. leo) (Mazák, 1981; Haas et al., 2005). En promedio, el león americano (Panthera atrox) del Pleistoceno tardío de Norteamérica es un 33\% más grande que un león africano actual (Van Valkenburgh et al., 2016; Bravo-Cuevas et al., 2016b). Por otra parte, los félidos del género Homotherium del Plioceno Pleistoceno de Norteamérica son de un tamaño similar al de un león africano. Por lo tanto, los félidos que generaron los rastros de Pie de Vaca fueron semejantes en tamaño a un tigre dientes de cimitarra y más pequeños que un león americano. Esto indicaría la presencia de macairodontinos en lo que ahora es la región centro-meridional del estado de Puebla durante el Cenozoico tardío. Cabe señalar que se ha propuesto la presencia de este grupo de félidos a partir de huellas en otras localidades del Neógeno tardío del occidente de México (Rodríguez-de la Rosa et al., 2007; Rodríguez-de la Rosa y Guzmán-Gutiérrez, 2012). La orientación diferente entre el RF 1 y los RF 2 - RF 3, así como el traslape de estos rastros con otros producidos por camélidos, sugiere que los félidos que los produjeron pasaron en momentos diferentes. La trayectoria del RF 1 indicaría que el individuo que lo generó eventualmente cambió de dirección. Por su parte, la orientación paralela de los RF 2 y RF 3, así como una velocidad relacionada con un modo de progresión en caminata para los organismos asociados a estos rastros, son evidencias que conducen a considerar una pareja de félidos.

La organización social en grupos entre los grandes félidos actuales es poco frecuente. Con relación a esto, el león africano es el único que desarrolla una marcada organización social (Rudnai, 1973; Nowell y Jackson, 1996; Anton y Turner, 1997; Packer, 2006). Otros grandes félidos como los tigres muestran cierto grado de sociabilidad, los chitas suelen formar coaliciones temporales de machos jóvenes, mientras que los jaguares y leopardos suelen ser solitarios (Anton y Turner, 1997; Packer, 2006). Entre los félidos extintos se ha considerado que el tigre dientes de sable (Smilodon fatalis) del
Pleistoceno tardío de Norteamérica tuvo un estilo de vida sociable, formando grupos familiares constituidos por parejas monógamas (Salesa et al., 2006); asimismo, un alto porcentaje de individuos juveniles y adultos de esta especie encontrados en la localidad Rancho La Brea, sugiere que era social como los lobos y leones actuales (McCall et al., 2003; Salesa et al., 2006; Carbone et al., 2009). El león americano Panthera atrox se sugiere que pudo ser solitario o tal vez organizarse en parejas Jefferson, 2001; Salesa et al., 2006; Carbone et al., 2009; Bravo-Cuevas et al., 2016b). La información acerca de los macairodontinos del género Homotherium sugiere que eran solitarios, debido al escaso número de individuos encontrados en Rancho La Brea (Carbone et al., 2009).

Con base en lo anterior y en función de que el registro de huellas de félidos de Pie de Vaca testificaría de manera indirecta la presencia de un individuo (RF 1) y una pareja (RF 2 y RF 3) que transitaron en momentos diferentes la zona, se propone un comportamiento semejante al que se ha considerado para el león americano y el tigre dientes de cimitarra.

Se sabe que los leones africanos y los tigres en caminata alcanzan velocidades promedio de 1.4 $\mathrm{m} / \mathrm{s}$ y $1.2 \mathrm{~m} / \mathrm{s}$ respectivamente. Las modalidades de trote y galope son raras y poco frecuentes, aunque leones en trote muestran velocidades de alrededor $2 \mathrm{~m} / \mathrm{s}$ (Rudnai, 1973). Anton et al. (2004) consideraron que la velocidad de los félidos que produjeron algunos de los rastros referidos a Felipeda lynxi y F. paroula del Mioceno de España, se asociaría a organismos en trote con una velocidad promedio de $1.3 \mathrm{~m} / \mathrm{s}$.

Con base en esto, la velocidad promedio de los félidos de Pie de Vaca $(1.8 \mathrm{~m} / \mathrm{s})$ se asociaría a una caminata rápida o trote.

\section{Conclusiones}

Se describe el conjunto de huellas de camélidos y félidos de la localidad Pie de Vaca en el estado de Puebla, centro de México; las de camélidos 
fueron designadas a la icnoespecie Lamaichnum guanicoe en atención a su tamaño y se sugiere que fueron producidas por algún integrante del género Hemiauchenia que fue la llama más común del Cenozoico tardío de México. Por su parte, las huellas de félidos fueron asignadas a la morfofamilia Felipedidae; posiblemente fueron producidas por alguna forma de talla mediana a grande como un macairodontino o un panterino. El modo de progresión de los camélidos y félidos se relaciona con organismos en caminata relativamente rápida y desplazándose a una velocidad inferior a los $4 \mathrm{~m} / \mathrm{s}$. La distribución y las direcciones de los rastros de camélidos, así como la velocidad y modalidad de progresión propuestas para los individuos que los generaron, indican un comportamiento gregario asociado a cierta organización social, tal vez tropas de llamas. En el caso de los félidos se propone un comportamiento solitario y/o en pareja, como el que se observa en la mayoría de los grandes félidos actuales.

\section{Agradecimientos}

La localidad Pie de Vaca se ha conservado hasta la actualidad gracias al interés y dedicación de la familia Aranguthy, quienes son los dueños del predio en el que se encuentra el afloramiento. Agradecemos especialmente a Don Félix Aranguthy por su siempre valiosa colaboración durante el desarrollo del estudio; asimismo, al biólogo Hugo Castro Azuara por su comprometida labor en la conservación del sitio. Al doctor Eduardo Jiménez Hidalgo (UMARPuerto Escondido, Oaxaca), al doctor Spencer G. Lucas (NMMNH), al Maestro en Ciencias Daniel Navarro (Museo de Paleontología, Facultad de Ciencias, UNAM) y al Biólogo Mariano Germán Arregui (Y-TEC/CONICET, Argentina) cuyos comentarios durante la revisión del manuscrito ayudaron en la mejora del mismo.

\section{Referencias}

Alexander, R.McN., 1976, Estimates of speeds of dinosaurs: Nature, 261, 129-130.

Alexander, R.McN., 2006, Dinosaur biomechanics: Proceedings of the Royal Society B, 273, 1849-1855.

Anton, M., Turner, A., 1997, The big cats and their fossil relatives: An illustrated guide to their evolution and natural history: New York, Columbia University Press, 234 p.

Anton, M., López, G., Santamaria, R., 2004, Carnivore Trackways from the Miocene Site of Salinas de Añana (Alava, Spain): Ichnos, $11,371-384$.

Applegate, S.P., Espinosa-Arrubarrena, L., LópezNeri, P., 1984, Las calizas litográficas de la Cantera Tlayúa en Tepexi de Rodríguez, Puebla, México: México, D.F., Universidad Nacional Autónoma de México, Instituto de Geología, Séptima Convención Geológica Nacional, Libreto Guía de la Excursión, 83 p.

Aramayo, S.A., de Bianco, T.M., 1987, Hallazgo de una icnofauna continental (Pleistoceno tardío) en la localidad de Pehuen-Co (Partido de Coronel Rosales), Provincia de Buenos Aires, Argentina, en IV Congreso Latinoamericano de Paleontología, Parte II, Carnivora, Artiodactyla y Aves: Santa Cruz de la Sierra, Bolivia, Asociación Boliviana de Paleontología, I, 532-547.

Aramayo, S.A., de Bianco, T.M., Bastianelli, N.V., Melchor, R.N., 2015, Pehuen Co: Updated taxonomic review of a late Pleistocene ichnological site in Argentina: Palaeogeography, Palaeoclimatology, Palaeoecology, 439, 144-165. 
Beraldi-Campesi, H., Cevallos-Ferriz, S.R.S., Centeno-García, E., Arenas-Abad, C., Fernández. L.P., 2006, Sedimentology and paleoecology of an Eocene-Oligocene alluvial-lacustrine arid system, Southern Mexico: Sedimentary Geology, 191, 227-254.

Bonacic, C., Franklin, W. L., 2006, Camels and llamas, en Macdonald, D.W. (ed.), The Princeton Encyclopedia of Mammals: China, Princeton University Press, 718-721.

Bravo-Cuevas, V.M., Jiménez-Hidalgo, E., Cuevas-Ruiz, G.E., Cabral-Perdomo, M.A., 2012, A small camelid Hemiauchenia from the Late Pleistocene of Hidalgo, central Mexico: Acta Palaeontologica Polonica, 57, 497-508.

Bravo-Cuevas, V. M., Arroyo-Cabrales, J., Priego-Vargas, J., 2016a, The record of camelids (Artiodactyla, Camelidae) from the Valsequillo Basin, Late Pleistocene of Puebla state, central Mexico: taxonomy, diet, and geographic distribution: Revista Brasileira de Paleontologia, 19, 243-258.

Bravo-Cuevas, V., M., Priego-Vargas, J., CabralPerdomo, M.A., Pineda Maldonado, M., 2016b, First occurrence of Panthera atrox (Felidae, Pantherinae) in the Mexican state of Hidalgo and a review of the record of felids from the Pleistocene of Mexico: Fossil Record, 19, 131-141.

Cabral-Perdomo, M.A., 1995, Los Icnofósiles de Vertebrados Terrestres del Terciario Tardío del área de Tepexi de Rodríguez, Estado de Puebla: México, D.F., Universidad Nacional Autónoma de México, tesis de licenciatura, $107 \mathrm{p}$.

Cabral-Perdomo, M.A., 2000, Pleistocene vertebrate tracks and traces from the Pie de Vaca Formation, en Sixtieth Annual Meeting, Society of Vertebrate Paleontology: México, D.F., México, Society of Vertebrate Paleontology, Guide Book of the Field Trips, 115-119.

Cabral-Perdomo, M.A., 2013, Icnofósiles de vertebrados terrestres del Cenozoico Tardío en el área de "Pie de Vaca", Tepexi de Rodríguex, Estado de Puebla: Paleontología Mexicana, 3, 51-58.

Carbone, C., Maddox, T., Funston, P.J., Mills, M.G.L., Grether, G.F., Van Valkenburgh, B., 2009, Parallels between playbacks and Pleistocene tar seeps suggest sociality in an extinct sabretooth cat, Smilodon: Biology Letters, 5, 81-85.

Castanera, D., Vila, B., Razzolini, N., dos Santos, V.F., Pascual, C., Canudo, J.I., 2012, Gregarious behaviour inferred from sauropod footprints in the Iberian Peninsula (resumen), en Martínez-Pérez, C., Furió, M., SantosCubedo, A., Poza, B. (eds.), Paleodiversity and Paleoecology of Iberian Ecosystems: Valencia, Sot de Chera, X Encuentro de Jóvenes Investigadores en Paleontología, $61-62$.

Collins, J.J., Stewart, I.N., 1993, Coupled nonlinear oscillators and the symmetries of animal gaits: Journal of Nonlinear Science, 3, 349-392.

Currier, M.J.P., 1983, Felis concolor: Mammalian Species, 200, 1-7.

Dávalos-Álvarez, O.G., Nieto-Samaniego, A.F., Alaniz-Álvarez, S.A., Martínez-Hernández, E., Ramírez-Arriaga, E., 2007, Estratigrafía cenozoica de la región de Tehuacán y su relación con el sector norte de la falla de Oaxaca: Revista Mexicana de Ciencias Geológicas, 24, 197-215. 
Diedrich, C.J., 2011, Late Pleistocene steppe lion Panthera leo spelaea (Goldfuss, 1810) footprints and bone records from open air sites in northern Germany - Evidence of hyena-lion antagonism and scavenging in Europe: Quaternary Science Reviews, 30, 1883-1906.

Franklin, W., 2011, Family Camelidae (Camels), en Wilson, D.E., Mittermeier, R.A. (eds.), Handbook of the mammals of the world, Hoofed Mammals: Barcelona, Lynx Edicions, 2, 206-247.

García-Ruiz, R., Goguitchaichvili, A., Cervantes-Solano, M., Cortés-Cortés, A., Morales-Contreras, J., Maciel-Peña, R., Rosas-Elguera, J., Macías-Vázquez, J.L., 2016, Secular variation and excursions of the Earth magnetic field during the PlioQuaternary: New paleomagnetic data from radiometrically dated lava flows of the Colima volcanic complex (western Mexico): Revista Mexicana de Ciencias Geológicas, $33,72-80$.

Haas, S.K., Haysen, V., Krausman, P.R., 2005, Panthera leo: Mammalian Species, 762, 1-11.

Hammer, Ø., Harper, D.A.T., Ryan, P.D., 2001, PAST: Paleontological statistics software package for education and data analysis: Palaeontologia Electronica, 4, 1-9.

Hemmer, H., 1972, Uncia uncia: Mammalian Species, 20, 1-5.

Henderson, D.M., 2003, Footprints, trackways, and hip heights of bipedal dinosaurs - testing hip height predictions with computer models: Ichnos, 10, 99-114.

Hulbert, R.C.jr., Webb, S.D., 2001, Artiodactyls, en Hulbert, R.C. (ed.), The fossil vertebrates of Florida: Florida, University Press of Florida, 242-279.
Jefferson, G.T., 2001, Naegele's giant jaguar, en Harris, J.M. (ed.), Rancho La Brea: Death Trap and Treasure Trove: Los Angeles, California, Terra, 2, 28-29.

Jefferson, G.T., Tejada-Flores, A., 1995, Late Blancan Acinonyx (Carnivora, Felidae) from the Vallecito Creek Local Fauna of AnzaBorrego Desert State Park, California: San Bernardino County Museum Quarterly, 42, $1-33$.

Jiménez-Hidalgo, E., Carranza-Castañeda, O., 2010, Blancan camelids from San Miguel de Allende, Guanajuato, central México: Journal of Paleontology, 84, 51-65.

Jiménez-Hidalgo, E., Carbot-Chanona, G., Castañeda-Posadas, C., 2015, El registro de mamíferos fósiles de Puebla, en CastañedaPosadas, C. (coord.), El registro paleobiológico del estado de Puebla: Puebla de Zaragoza, México, Benemérita Universidad Autónoma de Puebla, Escuela de Biología, 173-186.

Juárez-Arriaga, E. 2008. Pie de Vaca, formación (Grupo Balsas) Paleógeno (EocenoOligoceno): Léxico Estratigráfico de México, Servicio Geológico Mexicano, p. 1-6.

Kienapfel, K., Läbe, S., Preuschoft, H., 2014, Do tracks yield reliable information on gaits? Part 1: The case of horses: Fossil Record, 17, 59-67.

Kleiman, D.G., Eisenberg, J.F., 1973, Comparisons of canid and felid social systems from an evolutionary perspective: Animal Behaviour, 21, 637-659.

Krausman, P.R., Morales, S.M., 2005, Acinonyx jubatus: Mammalian Species, 771, 1-6.

Lange, I.M., 2002, Ice Age Mammals of North America: A Guide to the Big, the Hairy, and the Bizarre: Missoula, Montana, Mountain Press Publishing Company, 226 p. 
Liebenberg, L., 1990, A Field Guide to the Animal Tracks of Southern Africa: Sudáfrica, David Philip Publishers, 306 p.

Lockley, M.G., 1991. Tracking Dinosaurs: A New Look at an Ancient World: New York, Cambridge University Press, 242 p.

Lockley, M.G., Bishop, W., 2014, New footprint evidence of avian and mammal activity from a Miocene site near Durango, Mexico, en Lockley, M.G., Lucas, S.G. (eds.), Fossil Footprints of Western North America: Albuquerque, New Mexico, New Mexico Museum of Natural History and Science Bulletin, 62, 501-507.

Lockley, M., Delgado, C.R., 2007, Tracking an ancient turkey: A preliminary report on a new Miocene ichnofauna from near Durango, Mexico, en Lucas, S.G., Spielmann, J.A., Lockley, M.G. (eds.), Cenozoic Vertebrate Tracks and Traces: Albuquerque, New Mexico, New Mexico Museum of Natural History nd Science Bulletin, 42, 67-72.

Lucas, S.G., Hunt, A.P., 2007, Ichnotaxonomy of camel footprints, en Lucas, S.G., Spielmann, J.A., Lockley, M.G. (eds.), Cenozoic Vertebrate Tracks and Traces: Albuquerque, New Mexico, New Mexico Museum of Natural History and Science Bulletin, 42, 155-168.

Martínez-Hernández, E., Ramírez-Arriaga, E., 1999, Palinoestratigrafía de la región de Tepexi de Rodríguez, Puebla, México implicaciones cronoestratigráficas: Revista Mexicana de Ciencias Geológicas, 16, 187-207.

Mazák, V., 1981, Panthera tigris: Mammalian Species, 152, 1-8.

McCall, S., Naples, V., Martin, L., 2003, Assessing behavior in extinct animals: Was Smilodon social?: Brain, Behavior and Evolution, 61, 159-164.
North American Commission on Stratigraphic Nomenclature (NACSN), 2005, North American Stratigraphic Code: AAPG Bulletin, 89, 1547-1591.

Nowell, K., Jackson, P., 1996, Wild cats: Status survey and conservation action plan: Gland, Switzerland, International Union for Conservation of Nature and Natural Resources, $382 \mathrm{p}$.

Oliva, C., Arregui, M., 2018, Mammalian Ichnopathology: a case study of Holartic Ungulates (Gomphotheriidae, Equidae, Camelidae) of the Late Pleistocene of South America. Ichnotaxomic implications: Boletín de la Sociedad Geológica Mexicana, 70(2), 417-447.

Packer, C., 2006, Lion, en Macdonald, D.W. (ed.), The Princeton Encyclopedia of Mammals: China, Princeton University Press, 628-633. Pantoja-Alor, J., Malpica-Cruz, V.M., GalgueraRosas, G.A., 1989, Geología de los alrededores de la Cantera Tlayúa, Tepexi de Rodríguez, Puebla (resumen), en Memoria del Tercer Simposio sobre Geología Regional de México: México, D.F., Universidad Nacional Autónoma de México, Instituto de Geología, 61-69.

Pérez-Lorente, F., Herrero, C., Herrero, E., Montoya, P., 2009, Paracamelichnum Fumillensis n.ichnogen. n.ichnosp., Upper Miocene Camelidae Ichnites from the Hoya de la Sima site (Murcia, Spain): Ichnos, 16, 208-219.

Pfau, T., Hinton, E., Whitehead, C., WiktorowiczConroy, A., Hutchinson, J.R., 2011, Temporal gait parameters in the alpaca and the evolution of pacing and trotting locomotion in the Camelidae: Journal of Zoology, 283, 193-202.

Prothero, D.R., 2006, After the dinosaurs: The age of mammals: Bloomington, Indiana, Indiana University Press, 362 p. 
Raedeke, K.J., 1979, Population dynamics and socioecology of the guanaco (Lama guanicoe) of Magallanes, Chile: Washington, University of Washington, tesis doctoral, $427 \mathrm{p}$.

Remeika, P., Jefferson, G.T., Murray, L.K., 1995, Fossil vertebrate faunal list for the VallecitoFish Creek and Borrego-San Felipe Basins, Anza-Borrego Desert State Park and vicinity, California, en Remeika, P., Sturz, A. (eds.), Paleontology and Geology of the Western Salton Trough Detachment, AnzaBorrego Desert State Park, California: San Diego, California, San Diego Association of Geologists, Field trip guidebook and volume for the 1995 San Diego Association of Geologist's Field Trip to Anza-Borrego Desert State Park, I, 82-93.

Remeika, P., 2001, The Fish Creek Canyon ichnofauna: A Pliocene (Blancan) vertebrate footprint assemblage from Anza-Borrego Desert State Park, en Santucci, V.L., McGlelland, L. (eds.), Proceedings of the 6th Fossil Resource Conference: Colorado, Geological Resources Division, 55-75.

Remeika, P., 2006, Fossil footprints of AnzaBorrego, en Jefferson, G.T., Lindsay, L. (eds.), Fossil treasures of the Anza-Borrego Desert: The last seven million years: El Cajon, San Diego, Sunbelt Publications, 311-327.

Rock-Color Chart Committee, 2009, Geological Rock-Color chart: with genuine Munsell Color chips: Grand Rapids, Michigan, Geological Society of America - Munsell Color.

Rodríguez-de la Rosa, R.A., Guzmán-Gutiérrez, J.R., 2012, Huellas de aves y mamíferos del Neógeno de Jalisco, México: Paleontología Mexicana, 1, 147-158.

Rodríguez-de la Rosa, R.A., Aguillón-Martínez, M.C., López-Espinosa, J., Eberth, D.A.,
2004, The Fossil Record of Vertebrate Tracks in Mexico: Ichnos, 11, 27-37.

Rodríguez-de la Rosa, R.A., Polaco-Ramos, O.J., Aguilar, F.J., Guzmán-Gutiérrez, J.R., 2007, Footprints of Machairodontid Felids from the Late Tertiary of Central Mexico, en Díaz-Martínez, E., Rábano, I. (eds.), 4th European Meeting on the Palaeontology and Stratigraphy of Latin America: Madrid, España, Instituto Geológico y Minero de España, Cuadernos del Museo Geominero 8, 345-348.

Rudnai, J.A., 1973, The social life of the lion: A study of the behavior of wild lions (Panthera leo massaica [Newman]) in the Nairobi National Park, Kenya: Pennsylvania, Washington Square East Publishers, 122 p.

Salesa, M.J., Antón, M., Turner, A., Morales, J., 2006, Inferred behaviour and ecology of the primitive sabre-toothed cat Paramachairodus ogygia (Felidae, Machairodontinae) from the Late Miocene of Spain: Journal of Zoology, 268, 243-254.

Sarjeant, W.A.S., 1975, Fossil tracks and impressions of vertebrates, en Frey, R.W. (ed.), The Study of Trace Fossils: A Synthesis of Principles, Problems, and Procedures in Ichnology: New York, Springer-Verlag Berlin Heidelberg, 283-324.

Sarjeant, W.A.S., 1989, Ten Paleoichnological Commandments: a standardized procedure for the description of fossil vertebrate footprints, en Gillette, D.D., Lockley, M.G. (eds.), Dinosaur Tracks and Traces: New York, Cambridge University Press, 369-370.

Sarjeant, W.A.S., Langston, W.jr., 1994, Vertebrate footprints and invertebrate traces from the Chardonian (Late Eocene) of Trans-Pecos, Texas: Texas Memorial Museum Bulletin, 36, 1-86. 
Sarjeant, W.A.S., Reynolds, R.E., Kissell-Jones, M.M., 2002, Fossil Creodont and Carnivore Footprints from California, Nevada, and Wyoming, en Reynolds, R.E. (ed.), Between the Basins: Exploring the Western Mojave and Southern Basin and Range Province: Fullerton, California, California State University, Desert Studies Consortium - LSA Associates, 37-50.

Stein, A.B., Hayssen, V., 2013, Panthera pardus (Carnivora: Felidae): Mammalian Species, 45, 30-48.

Thulborn R.A., 1982, Speeds and gaits of dinosaurs: Palaeogeography, Palaeoclimatology, Palaeoecology, 38, 227-256.

Thulborn R.A., 1990, Dinosaur tracks: Londres, Chapman and Hall, 410 p.

Thulborn, R.A., Wade, M., 1984, Dinosaur trackways in the Winton formation (mid Cretaceous) of Queensland: Memoirs of the Queensland Museum, 21, 413-517.
Tomka, S.A., 1992, Vicuñas and Llamas: Parallels in Behavioral Ecology and Implications for the Domestication of Andean Camelids: Human Ecology, 20, 407-433.

Van der Sluijs, L., Gerken, M., Preuschoft, H., 2010, Comparative analysis of walking gaits in South American camelids: Journal of Zoology, 282, 291-299.

Van Valkenburgh, B., Hayward, M.W., Ripple, W.J., Meloro, C., Roth, V.L., 2016, The impact of large terrestrial carnivores on Pleistocene ecosystems: Proceedings of the National Academy of Sciences, 113, 862-867.

Vialov, O.S., 1966, Sledy zhiznedeiatel'nosti organizmow I ikh paleontogicheskoe znachenie: Kiev, Naukova Dumka, 219 p.

Webb, S.D., 1972, Locomotor evolution in camels: Forma et Functio, 5, 99-112.

Werderlin, L., 1985. Small Pleistocene Felines of North America: Journal of Vertebrate Paleontology, 5, 194-210. 